\title{
Biocrecimiento: síntesis dialógica entre \\ crecimiento, desarrollo y medio ambiente*
}

\section{Bio-growth: Dialogic Synthesis between Growth, Development, and the Environment}

\author{
Enrique Fuertes Grábalos**, Pablo Plou Lafuente***, \\ Carlos Gómez Bahillo****
}

Recibido: 2016-06-10 // Aprobado: 2016-09-20// Disponible en linea: 2017-01-30

Cómo citar este artículo: Fuertes Grábalos, E., Plou Lafuente, P. y Gómez Bahillo, C. (2017). Biocrecimiento: síntesis dialógica entre crecimiento, desarrollo y medioambiente. Ambiente y Desarrollo, 21(40), 43-56. https://doi.org/10.11144/Javeriana.ayd21-40.bsdc

doi:10.11144/Javeriana.ayd21-40.bsdc

\begin{abstract}
Resumen
La producción sin límites y la expansión del mercado globalizado está provocando crisis sistémicas que, a su vez, producen inestabilidad política y social a escala mundial, lo que muestra que la actual forma de crecimiento es insostenible a medio y largo plazo, por la dualización territorial y social que produce. En este artículo se reflexiona sobre ello. Se repasan las principales aportaciones sobre la relación hombre-naturaleza realizadas por los economistas clásicos y las propuestas que desde la economía y la sociología económica se están haciendo para proponer un modelo alternativo, en el cual el crecimiento económico no sea un fin en sí mismo. Se apuesta por un modelo de desarrollo respetuoso con el medio natural y solidario entre generaciones y pueblos.
\end{abstract}

Palabras clave: crecimiento; ecosistema; biocrecimiento; bioeconomía

Este artículo es de reflexión, presenta resultados de investigación desde una perspectiva analítica e interpretativa de los autores, con base en fuentes originales.

** Doctor en Sociología y Vicerrector de Investigación y Vinculación, Instituto Superior Tecnológico CrecermasProdas, Ecuador. Correo electrónico: enrifuer@yahoo.es

*** Licenciado en Ciencias Químicas y Vicerrector del Instituto Superior Tecnológico Crecermas-Prodas (Ecuador). Correo electrónico:pabloploulafuente@gmail.com

**** Doctor en Ciencias Políticas y Sociología y catedrático de Sociología, Facultad de Economía y Empresa, Universidad de Zaragoza, España. Correo electrónico: cgomez@unizar.es 


\begin{abstract}
Unrestrained production and the expansion of the globalized market are causing systemic crises, which in turn produce political and social instability on a global scale. This comes to show that the current form of growth is unsustainable in the mid and long term due to the social and territorial polarization it produces. This article reflects on said topic. We review the main contributions of classic economists on the relationship between man and nature and the proposals that are being made from economics and economic sociology to suggest an alternative model in which economic growth is not an end in itself. We opt for a development model respectful of the natural environment and supportive with generations and peoples.
\end{abstract}

Keywords: growth; ecosystem; bio-growth; bio-economics 


\section{Introducción: Biocrecimiento, ecodesarrollo, decrecimiento, crecimiento económico continuo y desarrollo sostenible}

La actividad transformadora y productiva del ser humano, el propio ser humano y las sociedades que conforma, son partes constitutivas de un sistema al que denominamos naturaleza (Gudynas, 2010). Es por este motivo que la actividad económica humana no puede razonablemente prescindir o trastocar, de una forma permanente o sostenida, los fundamentos, los procesos y la existencia misma de la naturaleza (Georgescu Rôetgen, 2003), por lo cual no es posible plantear que el crecimiento de la sociedad humana se pueda dar a costa de la destrucción sistemática de la naturaleza.

Esta simple idea es la que nos permite acercarnos al concepto de biocrecimiento. Biocrecer es crecer al mismo paso que progresa la naturaleza, dentro de los límites impuestos por su frágil y a un tiempo vigorosa capacidad de renovación y multiplicación, lo cual en la práctica implica apostar por un tipo de crecimiento que maximice el incremento de biomasa y biodiversidad y la generación de mínimos, o tal vez nulos, residuos, al mismo tiempo que buscamos aceptables rendimientos económicos. La actividad económica humana pasaría de buscar como único objetivo la maximización de los rendimientos económicos, a convertirse en una maximización multicriterio, en la cual se busca también, al mismo tiempo y de forma preferente, el incremento de la riqueza natural del planeta. Obviamente, en esta fórmula de multimaximización no se debe olvidar el progreso social y el enriquecimiento democrático de nuestras sociedades, aspecto que es inherente a todo verdadero progreso humano.

La propuesta del biocrecimiento supera la dualidad economía- naturaleza, de tal forma que ambas pasan a ser conceptos sinérgicos que multiplican la mutua capacidad de desarrollo y suponen, en definitiva, un planteamiento de ganar y ganar, lo cual conlleva el progreso natural y también mayores cotas de progreso de nuestras sociedades humanas.

No es objetivo de este artículo definir las propuestas de cómo, con el biocrecimiento, se podrían alcanzar estos objetivos. En este primer acercamiento se pretende analizar las principales referencias teóricas que desde el ámbito de la economía y de la sociología económica se han ido dando en las reflexiones sobre la relación hombre-naturaleza, y sobre la finitud de los recursos naturales y el deterioro medioambiental, que está afectando al bienestar y la calidad de vida de la población. Finalmente, se reflexiona sobre las aportaciones y propuestas alternativas; nos referimos no solo al biocrecimiento, sino también al denominado decrecimiento que, en definitiva, se plantean conseguir un modelo económico y social alternativo al hoy vigente.

El debate sobre crecimiento y desarrollo como su impacto medioambiental comienza a producirse en la década de los años setenta del siglo pasado, en las reuniones del Club de Roma, cuando se cuestionó el crecimiento como objetivo económico a escala mundial (Meadows, 1972). Los economistas clásicos buscaron nombres para seńalar un modelo de desarrollo económico que fuera más respetuoso con el entorno medioambiental, pero sin reducir los ritmos de producción y de expansión territorial; a ello se denominó ecodesarrollo (Organización de las Naciones Unidas [ONU], 1987). Frente a estas posturas aparecieron otras más radicales, como la de Daly (1973), para quién el desarrollo sostenible se fundamenta en el crecimiento cero, producido con una economía en estado estacionario, o la de Meadows (1972), quién cuestionó los conceptos de crecimiento y desarrollo utilizados por los economistas.

En el Primer Informe del Club de Roma, elaborado bajo la dirección de Meadows, y ante la limitación de los recursos naturales, la degradación del medio ambiente, la presión demográfica y el desigual grado de desarrollo entre unos países y otros, se consideró que el ritmo de crecimiento existente en las sociedades desarrolladas no era sostenible a largo plazo, y se propuso como objetivo alcanzar un estado de equilibrio duradero mediante un crecimiento sostenido o cero, y no aumentar la renta per cápita o producto nacional neto por habitante (ONU, 1987). 
A finales de los años ochenta, en el Informe Brundtland (ONU, 1987) se plantea ¿Cómo es posible el crecimiento económico y la protección medioambiental? (Gómez, 2005). La respuesta a esta cuestión se ha realizado desde diferentes perspectivas, considerando la relación existente entre crecimiento económico y utilización sin límites de los recursos naturales, o los efectos que este crecimiento está teniendo en la propia naturaleza por los impactos ambientales de las grandes acciones e intervenciones del hombre en la utilización del espacio, o analizando la vinculación entre la explosión demográfica y sus efectos en la biodiversidad y calidad ambiental, o relacionando el comercio global y la degradación medioambiental, etc.

Con crecimiento económico continuo nos referimos al incremento de la actividad económica de un país, y muy especialmente a la evolución de su producto interior bruto, mientras que, en el caso del desarrollo, se trata de la evolución ordenada y progresiva de la economía, orientada a la consecución de mayores niveles de vida y de bienestar de la población.

Por desarrollo sostenible se entiende, utilizando la definición hecha en el Informe Brundtland, y que fue nuevamente utilizada en la Declaración de Río de Janeiro (ONU, 1992), como un modelo de desarrollo que satisface las necesidades del presente sin condicionar las de las generaciones futuras, para lo cual se deben compatibilizar aspectos ambientales con los económicos y sociales, a partir de una perspectiva de solidaridad tanto inter como intrageneracional. El concepto de desarrollo sostenible tiene una implicación ambiental que considera el medio ambiente parte esencial de la vida humana, y que como tal debe ser objeto de protección especial. El deterioro medioambiental está asociado con el crecimiento económico y el consumo creciente de la población de las sociedades desarrolladas y de sus grupos sociales más selectivos, pero también con la pobreza de grupos y comunidades subdesarrolladas. Por lo tanto, el desarrollo sostenible requiere la viabilidad, a largo plazo, de la producción y el consumo mediante la utilización racional de los recursos disponibles.

La diferencia entre crecimiento y desarrollo económico radica en que, en el primer caso, nos estamos refiriendo al incremento de magnitudes macroeconómicas que manifiestan el dinamismo de la economía y el funcionamiento de su sistema productivo, mientras que por desarrollo entendemos la evolución progresiva de la economía que se manifiesta a través de la expansión del bienestar colectivo.

La Declaración de Río sobre el Medio Ambiente y el Desarrollo (ONU, 1992) reconoce el derecho de la población a una vida saludable y productiva, en armonía con la naturaleza, y el derecho de los estados a aprovechar los recursos naturales y su responsabilidad en la conservación del medio ambiente en su territorio. Se insiste en la responsabilidad sobre el mantenimiento de los ecosistemas y de las acciones que se realizan para evitar su degradación, destacando que, para mantener el medio ambiente a escala mundial, es necesario un sistema económico internacional que se compagine con el desarrollo sostenible.

Una de las posturas alternativas al modelo económico acumulativo global es el decrecimiento. El decrecimiento es una corriente de pensamiento político, económico y social que propone una disminución paulatina de la productividad y del consumo para restablecer una relación equilibrada con la naturaleza y entre los pueblos. Rechaza, por tanto, los planteamientos neoliberales y globalizadores basados en el incremento de la producción y la competitividad, al tiempo que promueve un cambio radical del actual sistema económico. La conservación medioambiental solo es posible si se reduce el actual nivel de productividad, que es el causante de la reducción de los recursos naturales y del deterioro del medio, por lo que se opone al modelo de desarrollo sostenible. Debido a ello, los partidarios del decrecimiento proponen una disminución del consumo y la producción controlada y racional, respetando los ecosistemas y buscando la mejora de la calidad de vida humana.

En todos los conceptos que hemos considerado, a excepción del biocrecimiento, observamos que, de alguna forma, se sobreentiende que crecimiento económico y naturaleza son conceptos, en cierto modo, antagónicos. Se diría que tan solo podemos aspirar a que la búsqueda del crecimiento o desarrollo se acompañe de medidas preventivas que minimicen los impactos negativos, que en todo caso 
se producirán, por lo cual naturaleza y progreso humano se nos presentan de hecho como conceptos enfrentados o contrapuestos. Es como si la humanidad aceptará conscientemente, aunque de forma no expresa, que su progreso está en últimas abocado al colapso de la especie, derivado de la afección ambiental que, de una u otra forma, el mismo progreso humano provoca, en un horizonte lo bastante lejano como para que por ello no nos sintamos presionados o afectados. Por eso, en todas las concepciones consideradas, a excepción del decrecimiento, y en cierta forma por innecesario en el biocrecimiento, se observa un punto en común: la fe ciega, a veces escasamente fundamentada en perspectivas científicas, y otras veces con tintes casi de certeza religiosa, en que la tecnología humana con su poder y capacidades extraordinarias y prometeicas siempre estará ahí, en condiciones de poder posponer el desastre

\section{Crecimiento y ambiente en la teoría socioeconómica}

\section{Crecimiento y ambiente en las teorías económicas clásicas}

Malthus (2000) plantea por primera vez la preocupación por la escasez de los recursos naturales, debido a que, según manifiesta, la producción de recursos alimenticios crece de forma aritmética y la población de forma geométrica, y centra su análisis en el recurso tierra, que considera finito. Por su parte, David Ricardo (2003) comparte la visión de que la economía debe llegar a un estado estacionario, conforme aumenta la demanda por parte de la población, por requerir su explotación mayores cantidades de capital y trabajo, pero no porque considere que los recursos sean finitos, sino porque la escasez relativa de un recurso, cualquiera que sea, se traduce en precios y costos crecientes, dado que el precio de los recursos es fijado por el último recurso de menor calidad o disponibilidad que ingresa en el proceso productivo. El progresivo aumento de los costos de producción terminaría por retrotraer todos los recursos financieros disponibles y, por tanto, la inversión dejaría de ser interesante para los propietarios del capital, lo que terminaría por generar un estado estacionario en la actividad económica. En consecuencia, Ricardo no plantea, al menos como algo necesario, que para alcanzar un estado estacionario de la economía sea necesario el agotamiento de los recursos.

Por otra parte, Adam Smith (2011) señala que la riqueza de un país depende del trabajo que realiza su población. El bienestar se consigue a través del crecimiento económico, por medio de la división del trabajo y de la libre competencia. El hombre es el motor del desarrollo, ya que a través de su buen hacer produce riqueza y acumulación de capital.

Frente al planteamiento de Adam Smith surgen fisiócratas como Quesnay o de Boisguillebert que remarcan la dependencia del hombre con respecto a la naturaleza (Bifani, 2007). Los fisiócratas, a mediados del siglo XVIII, ya utilizaron términos que hoy en día nos parecen familiares, puesto que proponían acrecentar la producción de riquezas renacientes (o quizás podrían haberlas denominado renovables), sin deteriorar los bienes de fondo (Naredo, 1993), como anticipando las propuestas del biocrecimiento.

John Stuart Mill (1997) concilia las visiones de Malthus y Ricardo dando más importancia a la escasez derivada del descenso de calidad y productividad de los recursos, y formula la ley más importante de la economía política: el efecto de escasez, que define cómo el incremento del costo de capital y trabajo por unidad de producción, debido a la incorporación al proceso productivo de recursos naturales de calidades inferiores o localizados desfavorablemente. Pero Mill señala el progreso de la civilización como fenómeno capaz de contrarrestar la tendencia a los rendimientos decrecientes.

Marx sostiene que la relación entre el hombre y la naturaleza es dialéctica y que, mientras existan hombres, la historia de la naturaleza y la historia de los hombres se condicionarán recíprocamente (Marx y Engels ([1845-1846] 2015). Sin embargo, también constata que, para el hombre, la naturaleza adquiere significado en tanto en cuanto tiene una utilidad para él, y además remarca el carácter social del concepto de naturaleza, el cual: "es una categoría social, esto es, siempre está socialmente condicionada [...], así como la relación de esa Naturaleza con el hombre” (Lukacs, 1969, p. 245). 
Este concepto de la naturaleza, un tanto subjetivo, y su carácter instrumental, probablemente llevan a Marx a no considerar un posible colapso como consecuencia de la escasez de recursos naturales. En este orden de cosas, desestima el problema poblacional planteado por Malthus, pues considera que las formas de producción condicionan el crecimiento poblacional. Marx (1851) rechaza los rendimientos decrecientes debido a la innovación y la tecnología.

Engels destaca el cortoplacismo que rige en los planteamientos productivos propios del sistema capitalista en relación con los efectos que produce en el medio ambiente: "Cuando los capitalistas se encuentran dedicados a la producción y el intercambio con vistas a la ganancia inmediata, sólo deben tenerse en cuenta en primer lugar los resultados más próximos e inmediatos” (Bifani, 2007, p. 23).

En cualquier caso, probablemente como reacción a la propuesta de los fisiócratas, Smith, Mill, Ricardo y Marx, entre otros, sostenían que la economía no tenía que ocuparse de lo físico. Los recursos naturales quedaban fuera del campo de la ciencia económica.

En los planteamientos de economistas clásicos como Stuart Mill, Ricardo y Smith, al no incorporarse de forma clara el problema del posible agotamiento de los recursos, se va desarrollando el fundamento teórico de lo que se denominará mecanicismo o neoclasicismo dentro de la ciencia económica, y sobre todo la consideración de los efectos del medioambiente en la economía como simples externalidades, lo que sin duda contrasta con los planteamientos que defienden la bioeconomía y el biocrecimiento, pues en estas disciplinas la naturaleza es el origen y sustento de toda posible actividad económica, lejos del antropocentrismo de las premisas económicas clásicas.

\section{Crecimiento y ambiente en el pensamiento neoclásico}

El pensamiento económico neoclásico parece más bien una reflexión en torno al objeto de la ciencia económica como disciplina científica. De ahí puede explicarse el esfuerzo casi obsesivo de los economistas neoclásicos por aislar de la discusión económica todas las interrelaciones con otras disciplinas de carácter empírico o social. Se construyen modelos y leyes económicas con base en situaciones supuestas y conceptos abstraídos de la realidad. La ciencia económica se centra en el funcionamiento del mercado, en su concepción más abstracta, preocupándose por los mecanismos que llevan a la fijación de precios y la asignación de recursos, así como por cuestiones financieras.

En este escenario, definir la relación que tiene la economía con el medioambiente o con la evolución de la sociedad resulta ajeno a la disciplina económica, motivo por el cual estas influencias se califican como externalidades, con lo que de nuevo nos alejamos de los planteamientos del biocrecimiento. Se busca el modelo matemático que rige los procesos económicos, considerando que los fenómenos económicos son iguales en toda circunstancia y lugar, y que todo fenómeno económico resulta reversible. Las reflexiones de la economía neoclásica se basan en el supuesto de la existencia de un mercado en el que se produce una libre concurrencia de la oferta y la demanda y un mecanismo transparente de fijación de precios e intereses. Este supuesto relaciona el pensamiento neoclásico con las tesis del capitalismo.

Por otra parte, el pensamiento neoclásico presupone el crecimiento económico como un contínuum sin límite. Pero el crecimiento económico continuo e ilimitado es un mito, un imposible. Esta aseveración resulta obvia si consideramos que el mundo que habitamos, soporte último de toda posible actividad humana, y por consiguiente de su actividad económica es, a su vez, limitado. No hay sistemas vitales en la tierra, y sin duda en el universo, que no tengan un crecimiento limitado.

Con respecto a la posibilidad de estados estacionarios de la economía, Marshall (2006) manifestaba que no existen razones para pensar que la humanidad se encuentre próxima a uno de tales estados. Keynes (1998), si bien admite la posibilidad de que se genere un estado estacionario, no lo considera inevitable, siempre y cuando se controle el crecimiento de la población, se regule adecuadamente el gasto público y la política fiscal, de acuerdo con el estado de la economía; asimismo, que se eviten 
las guerras y la ciencia guíe el desarrollo tecnológico. Hansen (1987) considera que la concatenación de crisis, cada vez más próximas en el tiempo, podría generar una escasez de inversiones posibles y, por ende, desembocar en un estado estacionario. En cualquier caso, dicho estado nunca parece estar relacionado con la escasez o agotamiento de los recursos naturales, como defiende la bioeconomía.

A finales del siglo XX, en el otro extremo conceptual al trabajo de los partidarios del crecimiento cero y de Meadows (1972), que se analizan más adelante, nos encontramos con Kahn y Simón (1984), quienes parten del supuesto de que por la propia lógica del crecimiento, la mayoría de los países terminarán por llegar al nivel de desarrollo de los países industrializados, siempre y cuando los avances tecnológicos permitan la introducción de nuevas prácticas agrícolas, se cambien los hábitos alimenticios de la población y se apliquen tecnologías para mejorar la producción de alimentos de alto contenido proteínico. Kahn, sin embargo, obvia en su análisis los impactos negativos medioambientales derivados del desarrollo tecnológico, y tampoco tiene en cuenta el previsible incremento del consumo derivado de los avances tecnológicos. Por otra parte, en su análisis no rechaza el agotamiento de los recursos minerales, tan solo pospone el colapso, en lo cual coincide con la bioeconomía (Bifani, 2007).

\section{Crecimiento estacionario o crecimiento cero}

A mitad del siglo XX, surge una nueva propuesta que no solo reconoce la posibilidad real del desencadenamiento de un estado estacionario de la economía y de la sociedad, originado por la escasez o agotamiento de los recursos naturales, sino que propone dicho estado estacionario como solución.

Kenneth E. Boulding (1945) constata que la humanidad vive en un sistema cerrado, con recursos limitados, espacios definidos y finitos, y que estos límites son cada vez más claros y perceptibles. Concibe el sistema económico actual como claramente expansivo y depredador, en tanto que Heilbroner (1970), con la misma orientación, anticipa que se ha superado el límite admisible de capacidad del planeta debido a la explosión de demográfica, y resalta el desequilibrio ecológico y las afecciones sobre el medio ambiente y la sociedad que se derivan de la tecnología (el incremento de dióxido de carbono, el exceso de agroquímicos y los impactos sociales en el sector rural ocasionados por la revolución verde), así como la pobreza y el hambre de una gran parte de la población, ante lo cual propone la necesidad de un crecimiento cero. Daly (1973), por su parte, sugiere que el flujo continuo que originan los recursos naturales de carácter renovable debería condicionar el flujo continuo e invariable de ingresos reales (Bifani, 2007).

Los debates y las propuestas del crecimiento cero terminan por inspirar a Meadows (1972) y los informes del Instituto Tecnológico de Massachussets que este economista dirigió para el Club de Roma (World III). Estos informes constituyen el primer intento serio de incorporar al pensamiento económico la idea de límites absolutos o escasez de los recursos naturales como un obstáculo al crecimiento. En dicho estudio se tienen en cuenta los límites absolutos de los recursos, el crecimiento exponencial de la población, los problemas de la contaminación y el deterioro del medio ambiente.

Meadows define dos submodelos: uno para los recursos naturales no renovables y otro para el sector agrícola. En el caso de los recursos minerales, determina que estos son limitados y augura su agotamiento definitivo, en relación con lo cual afirma también que el desarrollo tecnológico es incapaz de contrarrestar el incremento de costos crecientes en su explotación. En cuanto al sector agrícola, postula que la tierra agrícola arable es limitada y los costos para incorporar nuevas tierras son crecientes; además, los rendimientos de la actividad agrícola son decrecientes y la pérdida de terrenos de este tipo por la erosión, su utilización para otros fines (carreteras, construcciones habitacionales o industriales, etc.) y la pérdida de fertilidad y contaminación se incrementan de forma constante y significativa.

\section{Bioeconomía, decrecimiento y biocrecimiento}

Georgescu-Roegen $(1975,2003)$ formula una teoría económica alternativa: la bioeconomía. Esta es un esfuerzo por relacionar, e incluso subordinar, la economía con las ciencias naturales y 
sociales, y se fundamenta, principalmente, en la segunda ley de la termodinámica, aplicada a la transformación de la materia de los procesos productivos. Además, propone una cuarta ley de la termodinámica: "la materia disponible se degrada sin interrupción e irreversiblemente en materia no disponible" (Mosangini, 2007a, p. 10). Ni el reciclaje, ni la tecnología pueden revertir este proceso, ya que no existe un reciclaje gratuito, ni una industria sin residuos (Georgescu-Roegen, 2003). No podemos reciclar todos los residuos, ni tampoco la materia disipada que se ha perdido (Mosangini, 2007b). Y la tecnología tampoco es capaz de evitar la degradación final de la materia, tan solo permitirá producir lo mismo, con menos materia y energía y, al final, terminará provocando, por su mayor eficiencia, un mayor consumo y uso total de recursos y una mayor disipación de materia inutilizable.

Georgescu-Roegen (1994) concibe la historia económica de las civilizaciones como un proceso evolutivo (conectando así la economía con la biología), en el que el hombre ha ido incorporando progresivamente órganos exógenos o artificiales. Los órganos exosomáticos ideados por el hombre son adictivos, y no concibe la posibilidad de prescindir de ellos, y generan divergencias sociales y conflictos sociales. La utilidad de los órganos exosomáticos y su carácter externo los hace transferibles y, por tanto, se generan en torno a ellos procesos productivos, comercio y organizaciones complejas (Georgescu-Roegen, 1994).

La insostenibilidad del actual sistema económico no proviene de la futura escasez de energía, tanto como de la futura escasez de materia. Georgescu-Roegen ilustra de forma clara la insostenibilidad ecológica y social del actual sistema económico y propone minimizar nuestros remordimientos, más que maximizar los beneficios. Al desarrollar la bioeconomía, propone una economía que es ecológica y socialmente sostenible, y entiende el proceso económico como parte de nuestro mundo y, por tanto, sujeto a sus leyes y límites físicos.

Geogescu-Roegen y su bioeconomía han terminado por inspirar un movimiento que además de corriente de pensamiento, está siendo fuente de iniciativas sociales, de difusión crítica, denuncia e incluso puesta en práctica de sus postulados. Dicho movimiento se ha denominado decrecimiento.

El decrecimiento asume los análisis de la bioeconomía y postula la necesidad de romper con la lógica del crecimiento imparable, planteando la conveniencia de que la economía decrezca, sobre todo en los países del norte, como única opción para conseguir un sistema económico y social sostenible, enfocado en lograr mayores niveles de felicidad para los seres humanos (Taibo, 2015). Es importante que se consolide una sensibilización social acerca del deterioro medioambiental producido por la humanidad y sus efectos en las condiciones de vida próximas (Taibo, 2009).

Mosangini (2007b, 2012) considera que el decrecimiento permite analizar la crisis sistémica que el capitalismo global ha generado, y propone el concepto de deuda del crecimiento para compensar los impactos negativos que este proceso ha ocasionado en el sur por el crecimiento ilimitado del norte, y que son de carácter ecológico, social, cultural, histórico, económico, financiero, etc.

García Camarero (2009) describe, a través de unas premisas, la propuesta política (Programa de las $8 \mathrm{R}$ ) en torno al decrecimiento, inspirado en la propuesta de Osvaldo Pieroni en el Forum Alternativo de Río en 1992: Revaluar, Recontextualizar, Reestructurar, Relocalizar, Redistribuir, Reducir, Reutilizar y Reciclar.

También podríamos añadir como propuestas mencionadas entre los autores relacionados con el decrecimiento: la agricultura agroecológica, la producción artesanal, la desindustrialización, la apuesta por modelos de transporte locales y públicos, el neorruralismo y la desurbanización, la economía solidaria, la austeridad y la simplicidad voluntaria, los intercambios no monetarizados, el comercio justo, la banca ética, el consumo crítico, etc.

El biocrecimiento toma, al igual que el decrecimiento, todo el armazón conceptual de la bioeconomía, pero su propuesta principal no es decrecer la economía, más bien es hacerla crecer de forma 
sinérgica y acompasada con el ritmo de desarrollo de la naturaleza, al mismo tiempo que se produce una desmaterialización progresiva de la economía y el progreso social.

\section{Discusión: La bioeconomía y el decrecimiento como alternativa al mode- lo de crecimiento poscapitalista}

La economía es un subsistema dentro del sistema social, constituido por personas, instituciones, organizaciones públicas y privadas, Estado, recursos naturales, infraestructuras, etc., y por las relaciones existentes entre todos estos elementos para participar en la producción y distribución de bienes y servicios destinados a la sociedad humana. Por tanto, no puede estar al margen de las leyes que rigen los demás procesos de carácter social y, por extensión, los de carácter natural. Lo que implica que no puede haber sostenibilidad económica, verdaderamente, si no conseguimos una sostenibilidad social y ambiental.

Sin embargo, el vínculo entre el subsistema economía y el sistema que lo contiene — naturaleza—, no ha sido suficientemente tratado por la ciencia económica ortodoxa. Los tratados de economía habitualmente ignoran que la economía de nuestras sociedades es tan solo una parte de la economía ambiental o economía de los ecosistemas, y que, por tanto, en ningún momento las leyes que rigen la economía social o humana pueden llegar a contradecir las leyes que rigen la economía ambiental. En un alarde de antropocentrismo, hemos llegado a pretender incluir la autoadministración de los ecosistemas como un subsistema de nuestra economía humana, a la que denominamos economía de los recursos naturales. De cualquier forma, no se puede negar el papel que en su día desempeńó el antropocentrismo en el desarrollo científico y la modernización de las sociedades, aunque hoy en día nos impida reconocer los impactos que hemos generado en nuestro medio natural.

Si analizamos la economía humana desde la perspectiva de la economía ambiental, nos damos cuenta, en primer lugar, de que habitualmente en nuestros análisis económicos despreciamos o ignoramos los principales inputs y outputs. Por ejemplo, el calor recibido por nuestro astro rey; la luz; el oxígeno que nos proporciona la atmósfera para la obtención de energía para la supervivencia y los procesos vitales; la información recibida de nuestros padres y de nuestros ancestros comunes: hombres, homínidos, mamíferos, animales y todos los seres vivos; la energía y el tiempo entregados por nosotros y nuestros padres; el acervo de conocimientos y destrezas recibidos por nuestra cultura y los derechos que adquirimos por pertenecer a una sociedad (Martínez, 2002).

Todos estos bienes nos permiten administrar adecuadamente nuestra supervivencia, mejor que el dinero que usamos. Descubrir la limitación de la economía para explicar nuestra subsistencia y la administración y gestión de nuestras vidas, es uno de los aspectos que podemos destacar como novedad dentro de lo que es la herencia cultural económica.

En el siglo XX, la economía pretendía ser capaz de explicar al ser humano y el funcionamiento de la sociedad humana en su totalidad, y resultaba difícil discernir lo que no era un producto o consecuencia última de ella (Carpintero, 2005; 2006). Hoy en día, comprendemos que aquello era una simplificación, dado que el ser humano se entiende ahora como un producto o consecuencia de su integración con la naturaleza y, en menor medida, un producto de las relaciones económicas que se generan dentro de la sociedad humana.

En lo que respecta a la economía clásica y neoclásica debemos, en primer lugar, resaltar que esta adolece de rigor en su primera y más fundamental premisa: que el mercado es libre, y, por tanto, sus leyes en realidad son meros artificios mecanicistas que difícilmente pueden ajustarse a la realidad de los sistemas socioeconómicos.

Pero, descendiendo a las realidades desde el mundo de las abstracciones, se constata que el mercado libre no existe, ni existirá. En la práctica de los mercados es fácil comprobar cómo la concurrencia perfecta 
es más bien la excepción, siendo la norma todo un conjunto de prácticas económicas, de merchandising o industriales que pretenden, por último, prostituir el mecanismo supuestamente libre e imparcial del libre mercado en la fijación de los precios y las condiciones de los productos que se comercializan.

A la empresa capitalista el propio sistema de mercado le lleva casi irrevocablemente a procurar, por todos los medios posibles, unos legales y otros, en algún caso, ilegales o inmorales, a corromper el fundamento mismo del sistema de mercado: la libre competencia. Por otra parte, los países o zonas industrializadas, conscientes de que los productores de materias primas normalmente se concentran en países del sur, y temiendo que estos sean capaces de organizarse y tener peso decisorio en la fijación de precios, crearán de forma consciente y premeditada relaciones de dependencia, fundadas muchas veces en actos de corrupción. Teniendo en cuenta esta situación, el actual sistema económico lleva inexorablemente a su propia corrupción, dejando a la parte más débil del sistema, los pequeños productores y los pequeños consumidores, totalmente desprotegida.

Por otra parte, esta presión extractivista a la que se somete a los países del sur supone daños ambientales muy difícilmente reparables, que ocasionan pérdidas económicas, por las tierras baldías que se generan, sobrecostos en salud por los problemas en la salud pública y costos culturales por la pérdida de culturas propias y ancestrales. Lamentablemente, en Latinoamérica, el modelo extractivista es asumido como receta para el desarrollo por la clase empresarial, política y dirigente, y hasta por gran parte de la población, lo que derivan en enormes e incalculables costos ambientales actuales y diferidos (Gudynas, 2011).

En la actividad económica, bajo los esquemas del neoclasicismo económico, nunca se podrían evitar los efectos adversos de la actividad económica sobre el medioambiente, puesto que la decisión última del hecho económico subyace en el individuo, y este no puede decidir de manera racional acerca de las consecuencias de sus actos, si, simplemente, no llega a conocer las consecuencias ambientales de su decisión en el momento que decide. Además, y teniendo en cuenta lo dicho, resulta obvio que la posibilidad del estado estacionario es muy remota para los economistas neoclásicos, puesto que la naturaleza se encuentra en el terreno de las realidades y no en el de las abstracciones.

Si consideramos la propuesta del crecimiento cero deberemos considerar que:

1. Es de muy difícil aplicación, porque, en primer lugar, se precisarían periodos considerables para que los flujos poblacionales lleguen a ser estacionarios, lo que supondría un sistema de control de la población extremadamente complejo, sin dejar de considerar las soluciones del control de población con respecto a los derechos humanos.

2. El crecimiento cero no evita el agotamiento de los recursos, sino que lo pospone.

3. Parece que la propuesta se ha inspirado en un claro nortecentrismo, desde aquellos que han logrado un nivel de consumo más que suficiente y pretenden el mantenimiento de su estado actual. Quizás, por esta razón, en este debate sobre el crecimiento cero no se considera la interacción existente entre crecimiento económico, desarrollo y distribución equitativa de los recursos y la producción (Bifani, 2007). Además, se centra la consecución del crecimiento cero en la oferta de los recursos naturales, y no en la cuestión de la demanda, lo que implicaría esfuerzos adicionales a los países productores de materias primas.

4. No hay razones para suponer que el progreso tecnológico se detenga.

Adicionalmente, el cambio de modelo económico que promueve el decrecimiento no es fácil, y para algunos pensadores resulta imposible en las circunstancias actuales, por la preminencia de la economía acumulativa, a no ser que se produzca una desconstrucción de la racionalidad económica, y sea sustituida por otra economía fundada en una racionalidad ambiental (Leff, 2008).

En nuestro criterio, debido a varias consideraciones, el término decrecimiento no es afortunado. En primer lugar, surge desde una perspectiva nortecentrista; en efecto, puede coincidir con la 
disminución del crecimiento, siempre y cuando consideremos la perspectiva de los países desarrollados del norte; pero si consideramos a los países en desarrollo del sur, la perspectiva del decrecimiento cambia, puesto que, antes que disminuir, las economías de estos países deberían saldar las deudas históricas, sociales, económicas y ecológicas que se han generado después de cientos de años de aprovechamiento de los recursos del sur. En segundo lugar, el término decrecimiento parece responder a la necesidad dialéctica de contraponer el concepto al neocapitalismo.

Por último, somos conscientes de que el biocrecimiento, formulado de la manera en la que lo hemos hecho, pudiera parecer una propuesta demagógica sin un sustento real y que no se puede aspirar razonablemente a conseguir maximizar al mismo tiempo objetivos económicos, sociales y ambientales. También podríamos resistirnos a aceptar que el modelo industrializado e intensivo de producción pueda ser tan eficiente o incluso menos eficiente que la "creación o poiesis" que planteamos en el caso del biocrecimiento. Sin embargo, en la actualidad contamos con datos concluyentes que nos demuestran la superioridad en productividad y rentabilidad, en numerosas situaciones, de un modelo menos intensivo y más integrado de producción. Por otra parte, se están desarrollando modelos matemáticos, considerando precisamente este aspecto, sobre la base de propuestas productivas en torno al biocrecimiento, que nos han permitido estudiar y analizar mediante programación multicriterio la posible sinergia y consecución simultanea de múltiples objetivos económicos, sociales y ambientales. Pese al éxito inicial de estas investigaciones preliminares, debemos reconocer que, hoy por hoy, el modelo de desarrollo que plantea el biocrecimiento aún es una propuesta teórica, de la que estamos comenzando a conocer sus alcances.

Por otra parte, existe otra diferencia entre decrecimiento y biocrecimiento; el primero es más un movimiento social que se ha implicado de forma directa en la transformación y propuesta social de avanzada, mientras el segundo se plantea como una propuesta técnica que considera la posibilidad de proponer y demostrar la existencia de un nuevo modelo de desarrollo más equilibrado.

\section{Conclusiones}

Las crisis sistémicas cíclicas que experimentan las sociedades avanzadas (Habermas 1975), y la del 2008 es un ejemplo de ello, cuestionan la racionalidad del crecimiento indiferenciado del sistema capitalista, ya que se está demostrando que la abundancia de producción no conduce necesariamente al bienestar, y que la economía no puede crecer de forma indefinida, a causa de los límites físicos de la propia naturaleza.

El principal problema de la humanidad no es ya el crecimiento, sino la distribución de bienes y recursos. Únicamente será posible salir de estas crisis cíclicas que vivimos, a través de unas estrategias globales de cambio a escala mundial, que establezcan un nuevo marco general de cooperación y una nueva actitud hacia la naturaleza y la utilización de sus recursos, basada en la armonía y no en la dominación (Gómez, 2011).

No se puede crecer si no es al mismo tiempo que el entorno natural; no podemos desarrollarnos si no es al mismo paso que la naturaleza. Tampoco se puede plantear un crecimiento que genere materia inservible y energía disipada de forma ineficiente. Llevamos, sin embargo, más de un centenar de años de desarrollismo jugando a la contra de la naturaleza; se empezó predando los bosques europeos y ahora se están predando gran parte de las reservas fósiles del subsuelo, almacenadas durante miles de años. Igualmente, se han predado bosques, mares y caladeros y la fertilidad de los mejores suelos. Lo peor de todo, se hace en aras del progreso, y lo que hasta ahora se ha conseguido son sociedades desiguales e insostenibles. Pero, ¿acaso es posible progresar fuera del o contra el progreso natural?

Es necesario romper los paradigmas que definen el actual modelo de desarrollo. Ni el modelo económico clásico, ni el neoclásico, ni tan siquiera el crecimiento cero o el desarrollo sostenible son capaces de plantear una propuesta capaz de resolver el reto más importante al que nos enfrentamos como especie: ¿̇seguimos considerando un desarrollo que se basa en la destrucción sistemática del 
entorno natural y, por tanto, abocado a la destrucción como especie o, por el contrario, estamos realmente dispuestos a limitar nuestro desarrollo a los ritmos de la naturaleza?

No podemos confiar ciegamente en que la tecnología prometeica, al final, nos resolverá los problemas, porque lo que está en juego, nuestra supervivencia, es demasiado valioso. Por otra parte, la tecnología tan solo podría hacer más eficientes los procesos productivos, y esta mayor eficiencia redunda en un mayor consumo y una mayor generación de residuos en términos absolutos.

Maeterlink (2008), quien admiraba el progreso alcanzado por los insectos sociales, las abejas y otros himenópteros, se llegaba a preguntar, si no serían posibles sociedades humanas más eficientes, sostenibles, equilibradas, basadas en procesos regulados social, cultural y políticamente?

La bioeconomía planteada por Nicolás Georgescu supone un aporte teórico sobre el que podemos construir los nuevos mimbres y parámetros de nuestro desarrollo, pues propugna por un desarrollo humano ligado a las leyes de la naturaleza, generador de mínimos o nulos desechos materiales, y basado en la capacidad de generación y renovación naturales.

En torno a la bioeconomía han surgido dos propuestas: una, el decrecimiento, que plantea una opción clara de disminuir los niveles actuales de consumo y utilización de recursos naturales, apostando por la reducción, el reciclaje y la reutilización, aun a costa de disminuir nuestro nivel de crecimiento; y la segunda, el biocrecimiento, que defiende un modelo de crecimiento alineado y sinérgico con el desarrollo de la naturaleza.

\section{Referencias}

Ariés, P. (2005). Décroissance ou barbarie. Villeurbanne: CEDEX, Editions Golias.

Ariés, P. (2008). La Décroissance: un nouveau projet politique. Villeurbanne: CEDEX, Editions Golias.

Ariés, P. (2009). Désobéir et grandir - Vers une société de décroissance. Montreal (Canadá): Editions Ecosociété.

Ariés, P. (2012). Décroissance ou récession, la décroissance vue de gauche. Lyon (France): Editions Parangon.

Barnett, H. J. y Morse, C. (2013 [1963]). Scarcity and growth: the economics of natural resource availability. s. 1.: Routledge.

Bifani, V. (2007). Medio ambiente y desarrollo. Guadalajara (México): Editorial Universitaria.

Bonaiuti, M. (2001). La teoria bioeconomica. La "nuova economia" di N. Georgescu-Roegen. Roma: Carocci editore.

Bonaiuti, M. (2003). Bioeconomia. Verso un'altra economia ecologicamente e socialmente sostenibile. Turín: Bollati Boringhieri editore.

Boulding, K. E. (1945). The consumption concept in economic theory. American Economic Review, 35(2), 1-14.

Carpintero, O. (2005). El desafío de la bioeconomía. Ecología Política, (30), 41-58.

Carpintero, O. (2006). La BioEconomía de Georgescu-Roegen. Mataró (Espańa): Montesinos.

Cheynet, V. (2008). Le choc de la décroissance. París: Ed. de Seuil.

Cheynet, V. (2014). Décroissance ou décadence. Vierzon : Editions Le Pas de Côté.

Daly, H. E. (1973). Toward a steady state economy. San Francisco: Freeman.

García Camarero, J. (2009). El crecimiento mata y genera crisis terminal. Madrid: Libros de la Catarata.

Georgescu-Roegen, N. (1975). Energía y mitos económicos. Información Comercial Española, (501), 94-122.

Georgescu-Roegen, N. (1994). ¿Qué puede enseñar a los economistas la termodinámica y la biología? En F. Aguilera Klink y V. Alcántara (Comp.), De la economía ambiental a la economía ecológica (pp. 303-319). Madrid, Fuhem y Barcelona: Icaria. 
Georgescu-Roegen, N. (2003). Ineguaglianza, limiti e crescita da un punto di vista bioeconomico. En N. Georgescu-Roegen y M. Bonaiuti (Ed.), Bioeconomia. Turín: Bollati Boringhieri.

Gómez, C. (2005). Crecimiento económico y desarrollo sostenible en el medio rural. ¿Utopía o realidad? Revista de Desarrollo Rural y Cooperativismo Agrario, (9), 9-20. Recuperado de https://www.reseachgate,net/ publication/28137321

Gómez, C. (2011). La globalización y el nuevo orden/desorden mundial. La crisis de 2008. Sociedad y Utopía. Revista de Ciencias Sociales, (37), 115-138.

Grinevald, J. (1980). Le Sens bioéconomique du développement humain: l'affaire Nicholas Georgescu-Roegen. Revue européenne des sciences sociales et Cahiers Vilfredo Pareto, 18(51), 59-75.

Gudynas, E. (2010). Imágenes, ideas y conceptos sobre la naturaleza en América Latina. En E. Montenegro (Ed.), Cultura y naturaleza (pp. 267-292). Bogotá: Jardín Botánico J. C. Mutis.

Habermas, J. (1975). Los problemas de legitimación del capitalismo tardio. Buenos Aires: Amorrortu.

Hansen, A. (1987 [1957]). Guia de Keynes. Buenos Aires: Vértice.

Heilbroner, R. (1970). Ecological Armageddon. The New York Review of Books, 14(8). Recuperado en mayo del 2016, de http://www.nybooks.com/articles/1970/04/23/ecological-armageddon/

Kahn, H. y Simón, J. L. (1984). The resourceful earth a response to global 2000. Oxford: Basil Blackwell.

Keynes, J. M. (1998 [1936]). Teoría general de la ocupación, el interés y el dinero. Madrid: Ediciones Aosta.

Latouche, S. (2009a). La apuesta por el decrecimiento: ¿cómo salir del imaginario dominante? Barcelona: Icaria.

Latouche, S. (2009b). Decrecimiento y posdesarrollo: el pensamiento creativo contra la economía del absurdo. Barcelona: Icaria.

Latouche, S. (2009c). Pequeño tratado de decrecimiento sereno. Barcelona: Icaria.

Latouche, S. (2011). La hora del decrecimiento. Barcelona: Octaedro.

Leff, E. (2008). Decrecimiento o desconstrucción de la economía: Hacia un mundo sustentable. Polis, Revista de la Universidad Bolivariana, 7(21), 81-90.

Lukacs, G. (1969). Historia y conciencia de clase. México: Grijalbo.

Maeterlink, M. (2008)[1938]. La vida de las abejas. Barcelona: Editorial Planeta.

Malthus, T. (2000 [1798]). Primer ensayo sobre la población. Madrid: Alianza Editorial.

Marshall, A. (2006 [1890]). Principios de economía. Madrid: Editorial Síntesis.

Martínez Coll, J. C. (2002). Las flechas. Economía del tiempo y de la información. Recuperado de http://www. eumed.net/flechas/indice.html

Marx, K. (1851, 7 de enero). Carta de Marx a Engels. Recuperado en marzo del 2016, de https://www.marxists. org/espanol/m-e/cartas/m1851-01-07.htm

Marx, K. y Engels, F. (2015 [1845-1846]). La ideología alemana. Madrid: Akal.

Meadows, D. H. (1972). Los limites del crecimiento. Informe al Club de Roma sobre el predicamento de la humanidad. México: Fondo de Cultura Económica.

Mill, J. S. (1997 [1824-1825]). Ensayos sobre algunas cuestiones disputadas en economía política. Madrid: Alianza Editorial.

Mosangini, G. (2007a). El decrecimiento, camino hacia la sostenibilidad. En Ecologistas en Acción. Recuperado en marzo del 2016, de http://www.ecologistasenaccion.es/article13381.html 
Mosangini, G. (2007b). Decrecimiento y cooperación internacional. Recuperado en febrero del 2016, de http:// www.rebelion.org/noticia.php?id=56547

Mosangini, G. (2012). Decrecimiento y justicia Norte-Sur. Barcelona: Icaria.

Naredo, J. M.(1993). ¿Qué pueden hacer los economistas para ocuparse de los recursos naturales? desde el sistema económico hacia la economía de los sistemas. Madrid: Siglo XXI Editores.

Organización de las Naciones Unidas (ONU, 1987). Informe Brundtland: Crecimiento económico y desarrollo sostenible una única cuestión. Comisión Mundial sobre Medio Ambiente y Desarrollo de Naciones Unidas.

Organización de las Naciones Unidas (ONU, 1992). Declaración de Rio sobre el Medio Ambiente y el Desarrollo. Conferencia de Naciones Unidas sobre Medio Ambiente y Desarrollo, Río de Janeiro, junio de 1992.

Polanyi, K. (1989). La gran transformación. Madrid: La Piqueta.

Ricardo, D. (2003 [1817]). Principios de economía politica y tributación. Madrid: Pirámide.

Rist, G., Rahnema, M. y Esteva, G. (1992). Le Nord perdu, Repères pour l'après-développement. Lausana (Suiza): Editions d'En Bas, coll. Forum du développement.

Smith, A. (2011 [1776]). La riqueza de las naciones: Libros I-II-III y selección de los libros IVy V(Investigación sobre la naturaleza y causas de la riqueza de las naciones). Madrid: Alianza Editorial.

Taibo, C. (2009). En defensa del decrecimiento. Sobre capitalismo, crisis y barbarie. Madrid: Los Libros de la Catarata.

Taibo, C. (2015). Decrecimiento, crisis, capitalismo. Bilbao: Editorial Universidad del País Vasco, Colección de Estudios Internacionales. 